\title{
Kind-Philipp-Symposium zu Ehren des Stifters Herrn Dr.-Ing. Walter Reiners und Verleihung des Kind-Philipp-Preises 2008
}

\author{
Kind-Philipp Symposion in Honor to the Trustor Dr.-Ing. Walter Reiners and Kind-Philipp Award 2008
}

\section{T. Klingebiel ${ }^{1}$ \\ U. Creutzig ${ }^{2}$ \\ G. Henze ${ }^{3}$ \\ M. Hertl ${ }^{4}$ \\ U. Göbel ${ }^{5}$}

${ }^{1}$ Vorsitzender der Gesellschaft für Pädiatrische Onkologie und Hämatologie

${ }^{2}$ Geschäftsführerin der Gesellschaft für Pädiatrische Onkologie und Hämatologie

${ }^{3}$ Direktor der Klinik für Pädiatrie m. S. Onkologie und Hämatologie, Charité - Universitätsmedizin Berlin

${ }^{4}$ Vorsitzender des Beirats der Kind-Philipp-Stiftung für Leukämieforschung

${ }^{5}$ Herausgeber Klinische Pädiatrie

Bibliografie

DOI 10.1055/s-0029-1239505

Klin Padiatr 2009; 221: 327-331

(c) Georg Thieme Verlag KG

Stuttgart · New York

ISSN 0300-8630

Korrespondenzadresse

Prof. Dr. Ursula Creutzig GPOH Geschäftsführung Hannover

Thea- Bähnisch-Weg 12

30657 Hannover

Tel.: +49/511/604 6404

Fax: +49/511/604 6677

ursula@creutzig.de
Die Kind-Philipp-Stiftung für Leukämieforschung [30] im Stifterverband für die Deutsche Wissenschaft [53] wurde 1972 von Herrn Dr.-Ing. Walter Reiners aus Mönchengladbach zur Förderung der Erforschung der Grundlagen von Leukämie und Krebs im Kindesalter eingerichtet, nachdem sein Sohn Philipp ( $\bullet$ Abb. 1) an einer akuten Leukämie verstorben war [24]. Die Kind-Philipp-Stiftung, seit 1980 fortgeführt von Herrn Jan Reiners, dem Bruder des verstorbenen Philipp Reiners, ist seitdem eine Förderinstitution in der deutschen Wissenschaftslandschaft, die regelmäßig und mit hohem Engagement Forschung auf dem Gebiet der Pädiatrischen Onkologie fördert [25,27].

Die Förderung ist besonders fokussiert auf junge Wissenschaftler und Ärzte. Hierzu werden jährlich bis zu drei Promotionsstipendien vergeben. Mit den Jahrestagungen der Kind-Philipp-Stiftung für Leukämieforschung in Wilsede/ Lüneburger Heide besteht ein Forum, das jungen Wissenschaftlern die Möglichkeit gibt, nicht nur ihre Forschungsergebnisse unter sachkundiger Leitung zu präsentieren und zu diskutieren, sondern auch überregionale Kooperationen aufzubauen $[28,29]$. Hinzu kommen die in unregelmäßigen Abständen stattfindenden international ausgerichteten Kind-Philipp-Expertentagungen mit festgelegten Schwerpunkten; so ist zuletzt die Immuntherapie onkologischer Erkrankungen das verbindende Thema gewesen [32]. Die KindPhilipp Strukturtagung der Gesellschaft für Pädiatrische Onkologie und Hämatologie (GPOH) und des Kompetenznetzes Pädiatrische Onkologie und Hämatologie $(\mathrm{KPOH})$ dient insbesondere dem jährlichen Diskussionsaustausch zur Studiendurchführung und zur Weiterentwicklung der Studienqualität $[5,12,23]$. Jedes Jahr wird auch der Kind-Philipp-Preis für pädiatrisch-onkologische Forschung ausgeschrieben, mit dem die beste Arbeit deutschsprachiger Autoren zur Erforschung von Leukämie und Krebs bei Kindern ausgezeichnet werden soll.

Herr Dr.-Ing. Walter Reiners wäre im letzten Jahr 100 Jahre alt geworden und die GPOH hat diesen Geburtstag zum Anlass genommen, auf der 73. wissenschaftlichen Halbjahrestagung der $\mathrm{GPOH}$ am 22. Mai 2009 in Berlin, ein 2-stündiges Symposium zu veranstalten, um Herrn Reiners' Engagement zu würdigen.

Herr Professor Dr. Michael Hertl, Vorsitzender der Kind-Philipp-Stiftung und Beiratsmitglied der ersten Stunde, eröffnete das Symposium und zeigte die Bedeutung der Förderung durch die Kind-Philipp-Stiftung für die pädiatrisch-onkologische Forschung auf. Er betonte den Stellenwert privater Initiativen für die Verbesserung der Be- handlung und Prognose der an Leukämie und Krebs erkrankten Kinder [26].

Sieben Wissenschaftler, die in früheren Jahren mit dem Kind-Philipp-Preis ausgezeichnet worden sind, haben im Anschluss ausgehend von ihrer Preisarbeit über den Fortgang ihrer Forschungsarbeit berichtet. Dabei referierten sie über die Entwicklung und die Erfolge, die sich für die Behandlung und Heilbarkeit von onkologisch/ hämatologischen Erkrankungen bei Kindern und Jugendlichen in den letzten dreißig Jahren ergeben haben.

Die Vorträge zeigten in eindrucksvoller Weise, wie die Preisträger zur Entwicklung unseres Fachgebiets beigetragen haben. Prof. Dr. Dr. h.c. Günter Henze (Berlin) gab eine Übersicht über drei Jahrzehnte der Behandlung der akuten lymphatischen Leukämie, Prof. Dr. Ursula Creutzig (Münster) eine entsprechende Anlayse über akute myeloische Leukämie und die Risikoanpassung der Therapie. Prof. Dr. Michael Debatin (Ulm) zeigte überzeugend, was die Apoptoseforschung zum Verständnis der Leukämien beigetragen hat; Prof. Dr. Thomas Lion (Wien) konnte am Beispiel der CML die Bedeutung der Erforschung der minimalen Resterkrankung illustrieren. Prof. Dr. Holger Christiansen (Leipzig) und Prof. Dr. Angelika Eggert (Essen) konnten fundiert die Erforschung von Signalwegen und molekularen Prognosemarkern bei soliden Tumoren erläutern. Das Symposium endete mit einem Vortrag von Prof. Dr. Stefan Rutkowski (Hamburg) über die Therapieentwicklung bei Hirntumoren.

Die Gesamtgruppe der früheren Preisträger seit 1972 ist in der $\bullet$ Tabelle 1 mit den gewürdigten Arbeiten aufgelistet.

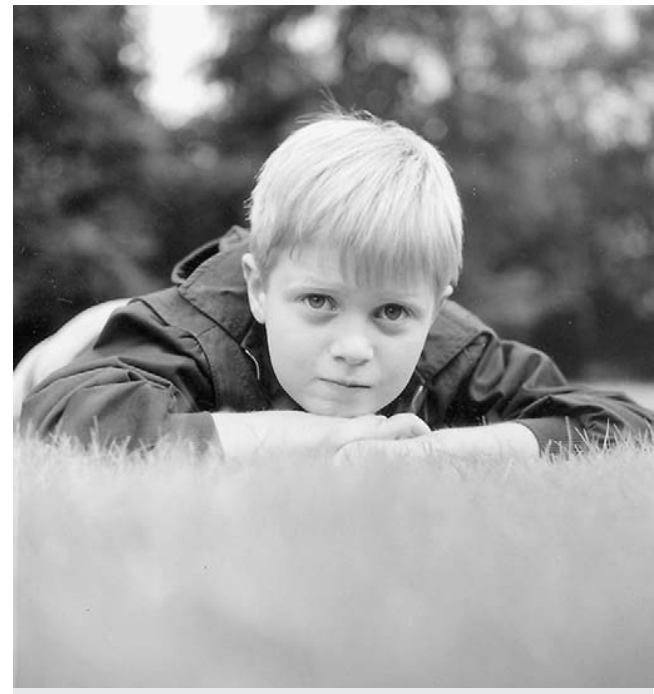

Abb. 1 Philipp Reiners. 
Die Stiftung ist ein hervorragendes Beispiel für großes und erfolgreiches bürgerschaftliches Engagement, das in einem ungewöhnlich effektiven Maß zum Gemeinwohl beiträgt. Dies betonte auch Frau Dr. Jutta Koch-Unterseher aus dem Berliner Senat für Wissenschaft und Forschung.

Am Ende des Kind-Philipp-Symposiums wurde der Kind-Philipp-Preis zum 30. Mal verliehen, wobei er in diesem Jahr erstmals dreigeteilt wurde. Der Stifterverband hat das gesamte Preisvolumen in diesem Jahr auf 15000 EUR aufgestockt. Zu drei gleichen Teilen wurde der Preis an die Arbeitsgruppe von Herrn Prof. Josef Vormoor mit Dr. Christoph le Viseur und Dr. Mark Hotfilder (Münster und Newcastle, UK) ( $\bullet$ Abb. 2), an Herrn Dr. Kaan Boztug (Hannover) ( $\bullet$ Abb. 3) und an Herrn Dr. Stefan Pfister (Heidelberg) ( $\bullet$ Abb. 3) vergeben. Somit vertraten die Preisträger die Bereiche Leukämieforschung, Tumorforschung und Erforschung der Immundefekte.

\section{Kind-Philipp-Preis-Arbeiten 2008}

$\nabla$

Arbeitsgruppe Prof. Dr. Josef Vormoor, Dr. Christoph le Viseur, Dr. Mark Hotfilder (Universitätsklinikum Münster, Newcastle University, UK). ( ( Abb. 2)

„Werden alle malignen Erkrankungen durch eine kleine Population unreifer Krebsstammzellen aufrechterhalten, wie myeloische Leukämien, oder besitzt die Mehrheit der malignen Zellen Stammzelleigenschaften?" Mit dieser Frage beschäftigte sich die Arbeitsgruppe und publizierte 2008 in Cancer Cell die Arbeit In childhood acute lymphoblastic leukemia, blasts at different stages of immunophenotypic maturation have stem cell properties [36].

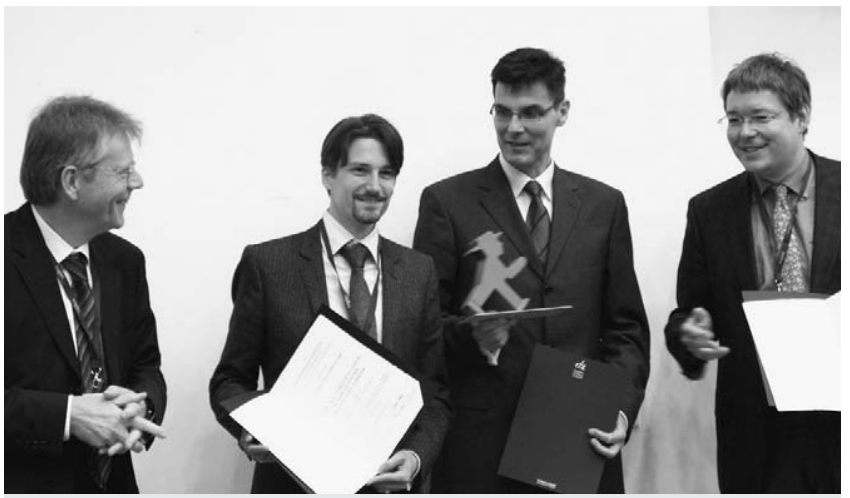

Abb. 2 Der Kind-Philipp-Preis 2008 wird der Arbeitsgruppe J. Vormoor übergeben (v.l.n.r.: Prof. Dr. T. Klingebiel, Dr. C. Le Viseur, Dr. M. Hotfilder, Prof. Dr. J. Vormoor).
Die Arbeit erlaubt einen Einblick in die Pathogenese der Leukämie im Kindesalter und zeigt neue Forschungsergebnisse auf, die zum Teil im Gegensatz zur herkömmlichen Meinung stehen. Durch die sehr sorgfältigen Untersuchungen mit einem neuen Mausmodell haben die Autoren erstmals gezeigt, dass das Stammzellkonzept nicht auf alle Leukämiearten übertragbar ist. Die Arbeit wurde am Universitätsklinikum Münster unter der Leitung von Prof. Dr. Vormoor erstellt. J. Vormoor absolvierte sein Medizinstudium in Münster und Newcastle, verbrachte seine post-doc Zeit in Kanada und wurde nach der Facharztausbildung in Münster Oberarzt. Er habilitierte 2006 und ist seit 2008 Inhaber des Sir James Spence Chair of Child Health an der Universität in Newcastle (UK).

C. le Viseur studierte Biologie in Mainz und Düsseldorf und promovierte in Münster. Zusätzlich absolvierte er eine Ausbildung zum Patentanwalt und ist nun Patentreferent in der freien Wirtschaft.

M. Hotfilder studierte ebenfalls Biologie in Köln und promovierte auch dort. Nach seiner Post-doc-Zeit in Münster wurde er Laborleiter der Pädiatrischen Hämatologie/Onkologie am dortigen Universitätsklinikum.

\section{Dr. Kaan Boztug (MH Hannover) (৫ Abb. 3; 4. v.l.)}

Die schwere kongenitale Neutropenie ( $\mathrm{SCN}$ ) ist eine seltene Erkrankung, mit deren zugrundeliegenden genetischen Defekten sich Dr. Boztugs Arbeit A syndrome with congenital neutropenia and mutations in G6PC3 beschäftigt [8].

Die Studie erklärt die verantwortliche molekulare Ätiologie und liefert wichtige Erkenntnisse für die Bedeutung Glucose-abhängiger Pathways für die Homöostase des endoplasmatischen Retikulums und die Kontrolle der Apoptose in myeloischen Zellen. Die Arbeit stellt eine wichtige Grundlage für ein verbessertes Verständnis der molekularen Prozesse dar, die der erhöhten Leukämogeneseneigung dieser Erkrankung zu Grunde liegen. K. Boztug studierte Medizin in Düsseldorf, Freiburg und London, verbrachte ein Forschungsjahr in den USA und erstellte seine Dissertation am Universitätsklinikum Freiburg. Seit 2005 arbeitet er in der Abteilung für Pädiatrische Hämatologie und Onkologie an der Medizinischen Hochschule Hannover.

\section{Dr. Stefan Pfister (Universitätsklinikum und DKFZ Heidelberg) (๑ Abb. 3; 2. v.r.)}

Für das Medulloblastom, die häufigste bösartige Hirntumorart im Kindesalter, ist es Dr. Pfisters Gruppe gelungen, ein einfaches, in der klinischen Praxis leicht umzusetzendes, molekulares Staging-System zu entwickeln. Der Titel der Preisarbeit lautet Outcome prediction in pediatric medulloblastoma based on DNA copy-number aberrations of chromosomes $6 q, 17 q$ and the MYC/MYCN loci [44]. Unter Zuhilfenahme molekularer Marker

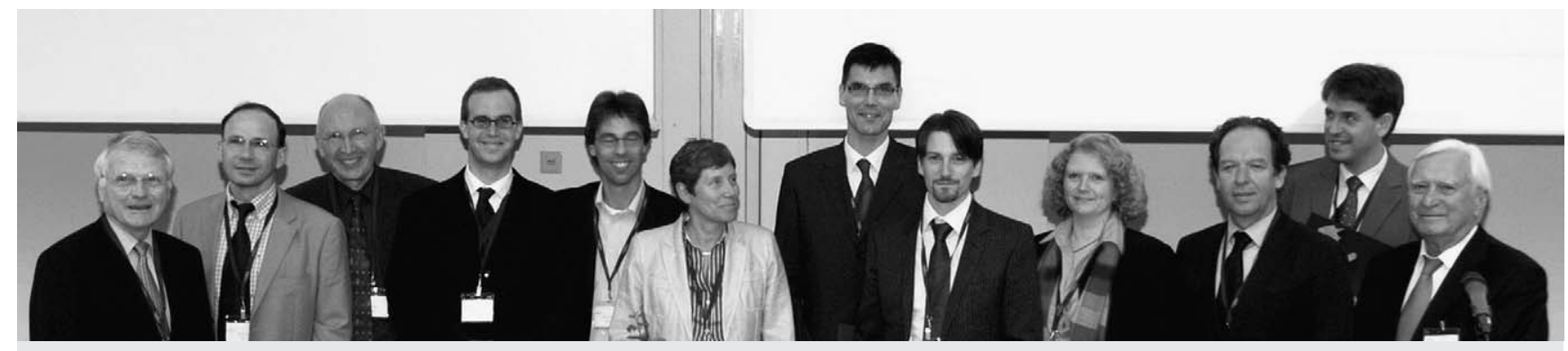

Abb. 3 Gruppenbild ehemaliger und aktueller Preisträger (v.I.n.r.: J. Reiners, Prof. Dr. T. Lion, Prof. Dr. Dr.h.c. G. Henze, Dr. K. Botzug, Prof. Dr. S. Rutkowski, Prof. Dr. U. Creutzig, Dr. M. Hotfilder, Dr. C. Le Viseur, Prof. Dr. A. Eggert, Prof. Dr. K.-M. Debatin, Dr. S. Pfister, Prof. M. Hertl). 
Tab. 1 Preisträger der Kind-Philipp-Stiftung für Leukämieforschung.

\begin{tabular}{|c|c|c|c|}
\hline Jahr & Preisträger & Thema & Literatur \\
\hline 1972 & $\begin{array}{l}\text { Norbert Kluge, Almut Knebel, Hans } \\
\text { Meldris, Bernd Weimann, Gerhard } \\
\text { Gaedicke, Shyam Dube, Wolfram } \\
\text { Ostertag, Göttingen }\end{array}$ & Die Polycythaemia vera als Modell zur Untersuchung der Entstehung von Leukämien & [43] \\
\hline 1973 & Hansjörg Riehm, Berlin & $\begin{array}{l}\text { Intensive kombinierte Induktionsbehandlung und ZNS-Bestrahlung der akuten } \\
\text { lymphoblastischen Leukämie des Kindes - Dokumentation und Bilanz nach drei Jahren }\end{array}$ & [49] \\
\hline 1975 & Karl Lennert, Kiel & $\begin{array}{l}\text { Klassifikation der Non-Hodgkin-Lymphome, insbesondere der hochgradig malignen } \\
\text { Lymphome des Kindesalters }\end{array}$ & [37] \\
\hline 1976 & Rudolf Eife, München & $\begin{array}{l}\text { Genuine und iatrogene Immunstörungen bei der akuten lymphoblastischen Leukämie } \\
\text { des Kindesalters }\end{array}$ & \\
\hline 1977 & $\begin{array}{l}\text { Prakash Chandra, Bernhard Korn- } \\
\text { huber, Horst Laube, Linda K. Steel, } \\
\text { Frankfurt a.M. }\end{array}$ & $\begin{array}{l}\text { Molekularbiologische Untersuchungen zur Virusatiologie eines präleukämischen } \\
\text { Syndroms im Kindesalter (Osteomyelofibrose) }\end{array}$ & {$[9,10]$} \\
\hline 1980 & $\begin{array}{l}\text { Günter Henze, Hans-Joachim Lan- } \\
\text { germann, Berlin }\end{array}$ & $\begin{array}{l}\text { Die Prognose der akuten lymphoblastischen Leukämie im Kindesalter in Abhängigkeit } \\
\text { von Initialbefunden und Therapie. Eine Analyse von drei Therapiestudien }\end{array}$ & {$[17,22]$} \\
\hline 1981 & Barbara Lau, München & $\begin{array}{l}\text { Differenzierungsvermögen und Proliferation von normalen und malignen Zellen } \\
\text { lympathischen Ursprungs }\end{array}$ & [35] \\
\hline 1982 & Thomas Graf, Heidelberg & Untersuchungen zum Mechanismus der virusinduzierten Leukämien & \\
\hline 1983 & $\begin{array}{l}\text { Claus Bartram und Gerard Grosveld, } \\
\text { Rotterdam }\end{array}$ & $\begin{array}{l}\text { Chronische myeloische Leukämie; Philadelphia-Translokation und humanes c-abl } \\
\text { Onkogen - Zusammenhänge im Lichte rekombinater DNS-Technologie }\end{array}$ & {$[4,41]$} \\
\hline 1984 & Michael Andreeff, New York & $\begin{array}{l}\text { Neue prognostische Faktoren der akuten Lymphoblastenleukämie des Kindesalters } \\
\text { und ihre Beziehung zu Zelldifferenzierung und Zellkinetik }\end{array}$ & \\
\hline 1986 & Karl Welte, Hannover & $\begin{array}{l}\text { Granulozytenkolonien stimulierender Faktor (G-CSF, Pluriopoietin), in vitro- und in } \\
\text { vivo-Effekte auf normale und leukämische myeloische Zellen }\end{array}$ & {$[21,54]$} \\
\hline 1987 & $\begin{array}{l}\text { Dusan Drahovsky (posthum) und } \\
\text { Thomas Boehm, Frankfurt a. M. }\end{array}$ & $\begin{array}{l}\text { Untersuchungen zur genetischen Heterogenität menschlicher Leukämien durch } \\
\text { Analyse der DNA-Arrangements von Immunglobulingenen, T-Zellrezeptorgenen und } \\
\text { zellulären Onkogenen }\end{array}$ & [6] \\
\hline 1988 & $\begin{array}{l}\text { Günther Schellong, Ursula Creutzig } \\
\text { und Jörg Ritter, Münster }\end{array}$ & $\begin{array}{l}\text { Akute myeloische Leukämie (AML) bei Kindern: Analyse der Therapiestudien AML- } \\
\text { BFM-78 und }-83 \text { als Basis für zukünftige Risiko-angepasste Behandlungsstrategien }\end{array}$ & {$[13,14]$} \\
\hline 1990 & $\begin{array}{l}\text { Peter H. Krammer, Klaus-Michael } \\
\text { Debatin, Heidelberg }\end{array}$ & $\begin{array}{l}\text { Wachstumskontrolle normaler und maligner Lymphozyten durch Induktion von } \\
\text { Apoptose }\end{array}$ & [15] \\
\hline 1991 & Lothar Schweigerer, Heidelberg & $\begin{array}{l}\text { Erhöhte MYCN-Expression im humanen Neuroblastom und Möglichkeiten der } \\
\text { therapeutischen Beeinflussung }\end{array}$ & {$[19,50]$} \\
\hline 1992 & Thomas Lion, Wien & Molekulargenetische Leukämiediagnostik - Nachweise minimaler Resterkrankung & {$[38,39]$} \\
\hline 1994 & Holger Christiansen, Gießen & $\begin{array}{l}\text { Tumorzellgenetische Eigenschaften und ihre medizinische Bedeutung beim Neuro- } \\
\text { blastom }\end{array}$ & [11] \\
\hline 1995 & $\begin{array}{l}\text { Markus Ollert, K. David, C. Schmitt, } \\
\text { Hamburg }\end{array}$ & Zytotoxische natürliche humane IgM Antikörper gegen Neuroblastomzellen & [42] \\
\hline 1996 & Ingeborg M. Ambros, Wien & Role of ploidy, chromosome 1 and Schwann cells in the maturation of neuroblastoma & [1] \\
\hline 1997 & Dietmar Lohmann, Essen & $\begin{array}{l}\text { Mutationsanalyse und prädikative Diagnostik bei erblichem und nicht-erblichem } \\
\text { Retinoblastom }\end{array}$ & [40] \\
\hline 1998 & Arndt Borkhardt, Gießen & Molekulargenetik von chromosomalen Aberrationen bei akuten Leukämien & [7] \\
\hline 2000 & Angelika Eggert, Essen & $\begin{array}{l}\text { Die prognostische und biologische Rolle von Neurotrophin-Rezeptoren bei soliden } \\
\text { Tumoren des Kindesalters }\end{array}$ & [16] \\
\hline 2001 & $\begin{array}{l}\text { Simone Fulda, Ulm } \\
\text { Stephan Ehl, Freiburg }\end{array}$ & $\begin{array}{l}\text { Apoptosemechanismen bei zytotoxischer Therapie } \\
\text { Aktivierung versus Tolerisierung von zytoxischen T-Zellen }\end{array}$ & {$[18,20]$} \\
\hline 2002 & Clemens Schmitt, Berlin & $\begin{array}{l}\text { Ein p53- und p16INK4a-kontrolliertes Seneszenz-Programm trägt zum Gesamtüber- } \\
\text { leben nach Chemotherapie bei }\end{array}$ & [47] \\
\hline \multirow[t]{2}{*}{2003} & Daniel Steinbach, Jena & $\begin{array}{l}\text { The multidrug resistance-associated protein } 3 \text { (MRP3) is associated with a poor out- } \\
\text { come in childhood ALL and may account for the worse prognosis in male patients and } \\
\text { T-cell immunophenotype }\end{array}$ & {$[51,52]$} \\
\hline & Peter Bader, Tübingen & $\begin{array}{l}\text { Increasing mixed chimerism is an important prognostic factor for unfavorable } \\
\text { outcome in children with ALL after allogeneic SCT - Possible role for pre-emptive } \\
\text { immunotherapy }\end{array}$ & {$[2,3]$} \\
\hline 2004 & $\begin{array}{l}\text { Stefan Rutkowski, Würzburg } \\
\text { Joachim Kühl, (posthum) }\end{array}$ & $\begin{array}{l}\text { Postoperative Chemotherapie bei Säuglingen und Kleinkindern mit Medulloblastom: } \\
\text { Ergebnisse der Studie HIT-SKK'92 }\end{array}$ & {$[45,46]$} \\
\hline \multirow[t]{2}{*}{2005} & Christian Kratz, Freiburg & $\begin{array}{l}\text { Germ line KRAS mutations encoding proteins with novel biochemical and functional } \\
\text { properties cause disorders of the Noonan syndrome spectrum }\end{array}$ & [33] \\
\hline & Alexander Schramm, Essen & $\begin{array}{l}\text { Anwendung von Genom- und Proteomanalysen zur Untersuchung der Tumorbiologie } \\
\text { und zur Therapieverbesserung des Neuroblastoms }\end{array}$ & [48] \\
\hline 2006 & Christoph Klein, Hannover & $\begin{array}{l}\text { HAX1 deficiency causes autosomal recessive severe congenital neutropenia-Kostmann } \\
\text { disease }\end{array}$ & {$[8,31]$} \\
\hline 2007 & Pablo Landgraf, Düsseldorf & A mammalian microRNA expression atlas based on small RNA library sequencing & [34] \\
\hline
\end{tabular}

Keine Preisverleihungen in den Jahren 1974, 1978, 1979, 1985, 1989, 1993, 1999 
gelang es der Arbeitsgruppe um S. Pfister, Patienten mit sehr schlechter Prognose zu identifizieren, die mithilfe der bislang verwendeten klinischen histo-pathologischen Marker als Standardrisikopatienten betrachtet worden wären. Darüber hinaus ist es gelungen, eine Gruppe von Good-risk-Patienten zu identifizieren, die möglicherweise von einer Reduktion der Therapie profitieren könnte.

S. Pfister studierte Medizin in Hamburg und verbrachte sein Praktisches Jahr in Basel und in Reutlingen, bevor er seine Dissertation an der Abteilung für pädiatrische Onkologie des Universitätsklinikums Tübingen ablegte. Seine Post-doc-Zeit verbrachte er am Dana-Farber-Institute, USA und am Deutschen Krebsforschungszentrum in Heidelberg. Er war Assistenzarzt in Mannheim und ist seit 2005 Mitarbeiter in der Abteilung Hämatologie, Onkologie und Immunologie des Universitätsklinikums Heidelberg.

Mit der 30. Verleihung des Kind-Philipp-Preises im Mai 2009 sind insgesamt 49 Kolleginnen und Kollegen mit dem in aller Welt hochangesehenen Wissenschaftspreis ausgezeichnet worden. ( Abb. 3) Das Kind-Philipp Symposium hat eindrücklich gezeigt, welche weiteren Forschungsaktivitäten durch den Preis angestoßen wurden und welche Verbesserungen für die Patienten daraus entstanden sind. Einen kleinen Einblick dazu geben die Folgepublikationen, die in der Tabelle zusätzlich zur jeweiligen Preisarbeit zitiert werden.

\section{Literatur}

1 Ambros IM, Zellner A, Roald B et al. Role of ploidy, chromosome 1p, and Schwann cells in the maturation of neuroblastoma. $\mathrm{N}$ Engl J Med 1996; 334: 1505-1511

2 Bader P, Kreyenberg H, Henze GH et al. Prognostic value of minimal residual disease quantification before allogeneic stem-cell transplantation in relapsed childhood acute lymphoblastic leukemia: The ALLREZ BFM Study Group. J Clin Oncol 2009; 27: 377-384

3 Bader $P$, Kreyenberg $H$, Hoelle $W$ et al. Increasing mixed chimerism is an important prognostic factor for unfavorable outcome in children with acute lymphoblastic leukemia after allogeneic stem-cell transplantation: possible role for pre-emptive immunotherapy? J Clin Oncol 2004; 22: 1696-1705

4 Bartram CR, de Klein A, Hagemeijer A et al. Translocation of c-ab1 oncogene correlates with the presence of a Philadelphia chromosome in chronic myelocytic leukaemia. Nature 1983; 306: 277-280

5 Berthold F, Bode G, Böcker A et al. Maßnahmen zur Qualitätssicherung für die stationäre kinderonkologische Versorgung. Klin Pädiatr 2006; 293-295

6 Boehm TL, Werle A, Ganser A et al. T cell receptor gamma chain variable gene rearrangements in acute lymphoblastic leukemias of $\mathrm{T}$ and B lineage. Eur J Immunol 1987; 17: 1593-1597

7 Borkhardt A, Harbott J, Lampert F. Biology and clinical significance of the TEL/AML1 rearrangement. Curr Opin Pediatr 1999; 11: 33-38

8 Boztug K, Appaswamy G, Ashikov A et al. A syndrome with congenital neutropenia and mutations in G6PC3. N Engl J Med 2009; 360: 32-43

9 Chandra P, Steel LK, Laube $H$ et al. Immunological Characterization of Reverse Transcriptase from Human Tumors: Evidence for SubgroupSpecific Interspecies antigenic Determinants on the Reverse Transcriptase Molecule. Cold Spring Harbor Conference on Cell Proliferation 1980; 7: 775-791

10 Chandra P, Steel LK, Laube $H$ et al. Expression of c-Type Viral Information in Tissues of Patients with Preleukemic Disorders: Myelofibrosis and granulocytic Sarcoma Associated with acute Myelomonocytic Leukemia (AMML) in children. Proceedings of the NATO ASI in: "Antiviral Mechanisms in the Control of Neoplasia" (Edt : P Chandra) 1979; 177-198

11 Christiansen H, Delattre O, Fuchs $S$ et al. Loss of the putative tumor suppressor-gene locus $1 \mathrm{p} 36$ as investigated by a PCR-assay and N-myc amplification in 48 neuroblastomas: results of the German Neuroblastoma Study Group. Prog Clin Biol Res 1994; 385: 19-25

12 Creutzig $U$, Henze $G$, Herold $R$ et al. Quality Management for Clinical Trials within the German Competence Network Paediatric Oncology and Haematology. Onkologie 2005; 28: 333-336
13 Creutzig U, Ritter J, Schellong G. Identification of two risk groups in childhood acute myelogenous leukemia after therapy intensification in the study AML-BFM-83 as compared with study AML-BFM-78. Blood 1990; 75: 1932-1940

14 Creutzig U, Zimmermann M, Ritter $J$ et al. Treatment strategies and long-term results in paediatric patients treated in four consecutive AML-BFM trials. Leukemia 2005; 19: 2030-2042

15 Debatin KM, Goldmann CK, Bamford $R$ et al. Monoclonal-antibodymediated apoptosis in adult T-cell leukaemia. Lancet 1990; 335: 497-500

16 Eggert A, Grotzer MA, Ikegaki $N$ et al. Expression of neurotrophin receptor TrkA inhibits angiogenesis in neuroblastoma. Med Pediatr Oncol 2000; 35: 569-572

17 Einsiedel HG, Von Stackelberg A, Hartmann R et al. Long-term outcome in children with relapsed ALL by risk-stratified salvage therapy: results of trial acute lymphoblastic leukemia-relapse study of the BerlinFrankfurt-Munster Group 87. J Clin Oncol 2005; 23: 7942-7950

18 Fakler M, Loeder S, Vogler $M$ et al. Small molecule XIAP inhibitors cooperate with TRAIL to induce apoptosis in childhood acute leukemia cells and overcome Bcl-2-mediated resistance. Blood 2009; 113: $1710-1722$

19 Fotsis T, Zhang Y, Pepper MS et al. The endogenous oestrogen metabolite 2-methoxyoestradiol inhibits angiogenesis and suppresses tumour growth. Nature 1994; 368: 237-239

20 Fulda S, Wick $W$, Weller $M$ et al. Smac agonists sensitize for Apo2L/T. Nat Med 2002; 8: 808-815

21 Germeshausen M, Ballmaier M, Welte K. Incidence of CSF3R mutations in severe congenital neutropenia and relevance for leukemogenesis: Results of a long-term survey. Blood 2007; 109: 93-99

22 Henze G, Langermann H-J, Lampert F et al. Die Studie zur Behandlung der akuten lymphoblastischen Leukämie 1971 - 1974 der Deutschen Arbeitsgemeinschaft für Leukämie-Forschung und -Behandlung im Kindesalter e.V. Analyse der prognostischen Bedeutung von Initialbefunden und Therapievarianten. Klin Padiatr 1979; 191: 8-20

23 Herold R, Reiche R, Creutzig $U$ et al. Stand und Bewertung der Strukturen und Ausstattung pädiatrisch-onkologisch-hämatologischer Kliniken. Klin Padiatr 2007; 219: 380-390

24 Hertl M. [Dr. Walter Reiners]. Klin Padiatr 1980; 192: 96-98

25 Hertl M. Kind-Philipp-Stiftung für Leukämieforschung 1972-1982. Klin Padiatr 1983; 195: 143-146

26 Hertl M. Die Bedeutung privater Initiativen für die Behandlung und Heilung der an Leukämie und Krebs erkrankten Kinder. Der Kinderarzt 1996; 27: 1287-1290

27 Jürgens H, Klingebiel T, Gobel U et al. Forschungsförderung für die Pädiatrische Onkologie und Hämatologie Supporting research in pediatric oncology and hematology. Klin Padiatr 2007; 219: 125-126

28 Kabisch H. Abstracts der XX. Jahrestagung der Kind-Philipp-Stiftung für Leukämieforschung. Wilsede/Lüneburger Heide. Klin Padiatr 2007; 219: $181-198$

29 Kabisch $H$. Abstracts der XX. Jahrestagung der Kind-Philipp-Stiftung für Leukämieforschung. Wilsede/Lüneburger Heide. Klin Padiatr 2008; 210: 198-214

30 Kind-Philipp-Stiftung für Leukämieforschung. www.kind-philipp-stiftung.de 2009

31 Klein C, Grudzien M, Appaswamy G et al. HAX1 deficiency causes autosomal recessive severe congenital neutropenia (Kostmann disease). Nat Genet 2007; 39: 86-92

32 Klingebiel T, Bader P, Hollatz G et al. Immunotherapy in children: Report from the Reisensburg-Symposium October 20-22, 2004 and recent advances. Klin Padiatr 2006; 218: 355-365

$33 \mathrm{Kratz} C P$, Niemeyer $C M$, Castleberry RP et al. The mutational spectrum of PTPN11 in juvenile myelomonocytic leukemia and Noonan syndrome/myeloproliferative disease. Blood 2005; 106: 2183-2185

34 Landgraf $P$, Rusu $M$, Sheridan $R$ et al. A mammalian microRNA expression atlas based on small RNA library sequencing. Cell 2007; 129: 1401-1414

35 Lau B, Jager G, Thiel E et al. Phenotypic changes in acute lymphoblastic leukemia cells of the common type in diffusion chambers. Leuk Res 1980; 4: 561-569

36 le Viseur C, Hotfilder M, Bomken S et al. In childhood acute lymphoblastic leukemia, blasts at different stages of immunophenotypic maturation have stem cell properties. Cancer Cell 2008; 14: 47-58

37 Lennert K, Stein H, Kaiserling E. Cytological and functional criteria for the classification of malignant lymphomata. Br J Cancer Suppl 1975; 2: $29-43$

38 Lion T. Monitoring of residual disease in chronic myelogenous leukemia by quantitative polymerase chain reaction and clinical decision making. Blood 1999; 94: 1486-1488 
39 Lion T, Henn T, Gaiger A et al. Early detection of relapse after bone marrow transplantation in patients with chronic myelogenous leukaemia. Lancet 1993; 341: 275-276

40 Lohmann DR, Gerick M, Brandt B et al. Constitutional RB1-gene mutations in patients with isolated unilateral retinoblastoma. Am J Hum Genet 1997; 61: 282-294

41 Morgan GJ, Hughes T, Janssen JW et al. Polymerase chain reaction for detection of residual leukaemia. Lancet 1989; 1: 928-929

42 Ollert MW, David K, Schmitt C et al. Normal human serum contains a natural IgM antibody cytotoxic for human neuroblastoma cells. Proc Natl Acad Sci USA 1996; 93: 4498-4503

43 Ostertag $W$, Melderis $H$, Steinheider $G$ et al. Synthesis of mouse haemoglobin and globin mRNA in leukaemic cell cultures. Nat New Biol 1972; 239: 231-234

44 Pfister S, Remke M, Benner A et al. Outcome prediction in pediatric medulloblastoma based on DNA copy-number aberrations of chromosomes $6 \mathrm{q}$ and $17 \mathrm{q}$ and the MYC and MYCN loci. J Clin Oncol 2009; 27: $1627-1636$

45 Rutkowski S, Bode U, Deinlein F et al. Treatment of early childhood medulloblastoma by postoperative chemotherapy alone. N Engl J Med 2005; 352: 978-986

46 Rutkowski S, Gerber NU, von Hoff K et al. Treatment of early childhood medulloblastoma by postoperative chemotherapy and deferred radiotherapy. Neuro Oncol 2009; 11: 201-210

47 Schmitt CA, Fridman JS, Yang $M$ et al. A senescence program controlled by p53 and p16INK4a contributes to the outcome of cancer therapy. Cell 2002; 109: 335-346
48 Schramm A, Schulte JH, Klein-Hitpass L et al. Prediction of clinical outcome and biological characterization of neuroblastoma by expression profiling. Oncogene 2005; 24: 7902-7912

49 Schrappe M, Reiter A, Zimmermann $M$ et al. Long-term results of four consecutive trials in childhood ALL performed by the ALL-BFM study group from 1981 to 1995 Berlin-Frankfurt-Munster. Leukemia 2000; 14: 2205-2222

50 Schweigerer L, Breit S, Wenzel A et al. Augmented MYCN expression advances the malignant phenotype of human neuroblastoma cells: evidence for induction of autocrine growth factor activity. Cancer Res 1990; 50: 4411-4416

51 Steinbach $D$, Legrand $O . A B C$ transporters and drug resistance in leukemia: was P-gp nothing but the first head of the Hydra? Leukemia 2007; 21: 1172-1176

52 Steinbach $D$, Wittig $S$, Cario $G$ et al. The multidrug resistance-associated protein 3 (MRP3) is associated with a poor outcome in childhood ALL and may account for the worse prognosis in male patients and T-cell immunophenotype. Blood 2003; 102: 4493-4498

53 Stifterverband für die Deutsche Wissenschaft. www.stifterverband.de 2009

54 Welte K, Platzer E, Lu L et al. Purification and biochemical characterization of human pluripotent hematopoietic colony-stimulating factor. Proc Natl Acad Sci USA 1985; 82: 1526-1530 